\title{
A DESIGN APPROACH USING BIO-GEOMETRY IN INTERIOR ARCHITECTURAL SPACES: Reference to Heal Attention Deficit Hyperactivity Disorder (ADHD)
}

\author{
Ihab Rashed \\ High Institute of Engineering, Sherouk Academy \\ Dina Howeidy \\ Architecture and Interior Design Department, Price Sultan University, Riyadh
}

\begin{abstract}
Since the beginning of the universe, people live in a middle of a huge amount of different types of energies which affect them, include what is useful, and what is harmful. The variation of these effects either direct or indirect was because of the role played by both designers architect and interior architect in the formation of voids. Both were responsible for the interior space design to avoid environmental pollution, and negative impacts to take the advantage of the positive energy in improving people performance in the interior architectural spaces, and the society in general. When we consider that at least $90 \%$ of our life is spent indoors, the significance of this sentence becomes apparent. ${ }^{1}$ Scientists have proved that the interior architectural space contains different types of energy. This energy has a negative and positive impact on human beings, some problems appeared like the absence of the concept "earth energy" and other environmental concepts associated with it, in addition to its impact on human health and performance in the interior architectural spaces, resulting in health problems for people in those spaces, Also the positive impact of bio-geometry on human health, through its application in the interior spaces to achieve a balance in the internal energy, thus helps in the treatment of many diseases. ${ }^{2}$
\end{abstract}

The main research aim is to use the principles and theories of Bio-Geometry in the interior architectural spaces, to achieve the best results to maintain human health, the efficiency of performance, and modify human behavior. In addition to formulate a clear framework and methodology as a design approach through which the designer either an architect or interior can deal with the negative energy in the interior architectural spaces using the science of BioGeometry, the paper followed the mentioned methodology to achieve the best results. This research Paper focuses on the importance of the science of biogeometry on human health as one of the application for the new science, the research will be applied on children who suffers from ADHD that considered as one of the new sciences that give more care to human health and performance, all results after testing the biogeometry will be statically examined by SPAA program and compare them all by the end to proof the ability of the new science to help in healing some health problems.

Key words: Bio-Geometry- Earth Energy-Geo-pathic stress-Attention Deficit Hyperactivity disorder (ADHD) -Bio-Energy- EMF: Electro Magnetic Field.

\footnotetext{
${ }^{1}$ Baker, Paula-and others-"A Healthy House"-2001,p.11

${ }^{2}$ Ibid.
} 
مع التقدم المتصل بعلم الطبيعة و الفيزياء ونظرياته مع أو اخر القرن العشرين، ظهرت مجمو عة من الأفكار و العلوم المتعلقة

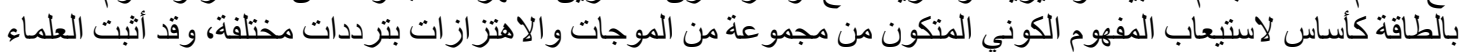

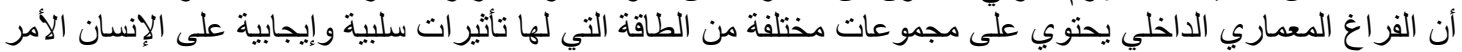

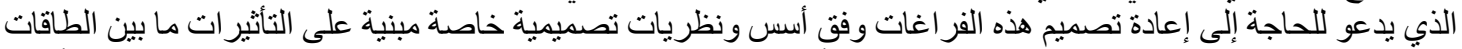

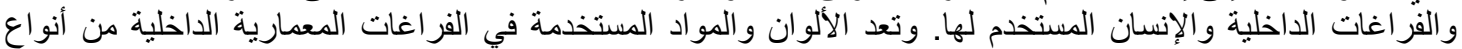

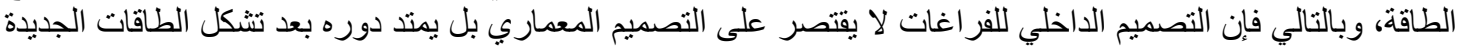

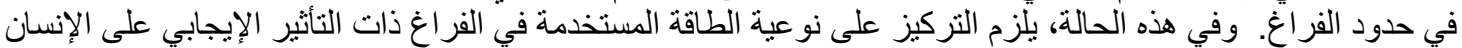

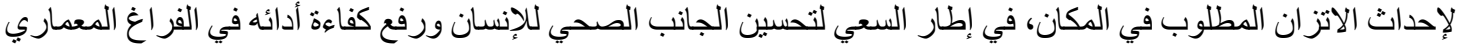

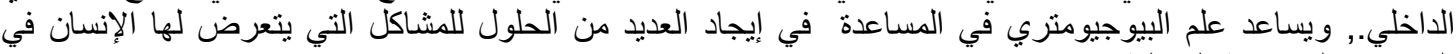

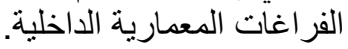

\section{INTRODUCTION}

Bio-Geometry is a science that deals with the Energy of Shape; it uses shapes, colors, motion, orientation and sound produce a vibrational quality that balances energy fields. Bio-Geometrical shapes are two or three-dimensional shapes specially designed to interact with the earth's energy fields to produce a balanced effect on multiple levels of biological systems. They were developed and patented by Dr. Ibrahim F. Karim, D.Sc. In Cairo, Egypt, during research since $1968 .^{3}$ It is also defined as a design language of geometric form, color, sound, and motion. The geometrical shapes are based on the science of micro-vibration physics, or radistezia. This science will create a new form of architecture that would enhance the human biological system and give a new meaning to the concept of building spaces. ${ }^{4}$

Bio-Geometry is the science that study effect of shapes and angles on the living organisms and their energy fields. It gives solutions to the negative harmful effects and enhances their positive effects. Bio-Geometry provides answers to the architectural pollution people suffer from, as well as the pollution of the technology (the use of electric, wireless and cellular devices in buildings). ${ }^{5}$

The interior energy system uses the senses and the energy centers (Chakras) to interact directly with all levels of energy in the environment. The peripheral energy systems in turn, are not completely closed to the outside either they are in constant exchange of information with the outside energy environment. ${ }^{6}$

This research will focus on the impact of Bio-geometry on students who are suffering from Attention-Defect Hyperactivity Disorder (ADHD) which can be defined as a group of behaviors found in many children and adults. People who have ADHD have trouble in paying attention at school, home or at work. They may be much more active and/or

\footnotetext{
${ }^{3}$ http://www.biogeometry.com/english/biogeo.php

${ }^{4}$ Dr. Ibrahim Karim-"What is energy-Back to the Future"-International Union of Architects ConferenceBibliotheca Alex.- Alexndrina-2002

${ }^{5}$ Dr. Ibrahim Karim ," Bio-Geometrical Energy Balancing" cd, 2002

${ }^{6}$ Ibrahim Karim-"Back To A future For Mankind"-publisher: Bio-geometry consultant centre2009,p.247
} 
impulsive than what is usual for their age. These behaviors contribute to significant problems in relationships, learning and behavior. For this reason, children who have ADHD are sometimes seen as being "Difficult" or as having behavior problems. ${ }^{7}$

The research will concentrate on applying the design approach on the case study to change children medical case "ADHD" in a specialized approved center HDC (Human Development Centre). This is to help in treating those cases in which designers' role can have a successful design approach for helping people to be cured from the pollutant interior environments.

\section{DESIGN APPROACH STEPS}

The development of science and the appearance of the new research there has been awareness and interest of the interpretation of many of the phenomena seen in life such as art, humanities, and religion. All are sciences that has the nature of the different quality is not measurable or quantitatively ranking and scientifically therefore was necessary to emergence of new concepts that can explain the phenomena of the quality of the universe. These following are concepts related to the Bio-Geometry science:

- Organized Energy

- Golden component at higher levels

- Ultra Violet component at higher levels

- Negative Green

-Energy Key

- Radistezia

- Harmonics

- Measure the Qualities and Quantities

To apply the design approach to the case study the research will go through the following steps:

Stimuli:

Four reasons were behind choosing the case study on ADHD:

- ADHD is a common behavioral disorder that affects about $8 \%$ to $10 \%$ of schoolage children. Boys are about three times more likely than girls to be diagnosed with.

- The reason(s) that causes negative impact on human body in the interior architectural space, such as Geo-pathic stress on children that causes ADHD and LD (Learning Disability).

- The children with this problem should be healed otherwise it will cause increasing in the number of criminals in society according to the latest studies.

- Clarification of the designer's role on interior space as a designer to help the children with (ADHD), and improve their performance using color therapy and bio-geometry cubes and shapes, in addition to windows designs, and interior

\footnotetext{
7 -http://www.adhd.com/index.html
} 
architectural space's energy balance, to achieve all requirements and needs of mental health.

Method:

The setting of the case study (Input data-Process (method) -Output data) will be through 4 stages of tests and results using the checklists as the following:

- -Stage 1: The base case without any edit in the interior space, and this is used as a scale to compare the results before and after using the Bio-Geometry, monitor the time and performance needed for development through diagnoses and final reports.

- -Stage 2: Depends on using BG energy cubes, shapes, colors, and records the student's performance according to the same limited time that was given to others in the other stages.

- -Stage 3: Depends on using BG energy cubes, shapes, colors, in addition to window designs, interior spaces energy balance, doors balancing energy, and records the student's performance according to the same limited time that was given to other stages.

- - Stage 4:Compare all final results.

\section{DESIGN APPROACH ANALYSIS}

Analysis for developing the Interior Architectural Space dealt with participants, study area, energy detecting tools and equipment, questionnaires, and observation. All stages will be done by the physicians, specialists in the HDC, and the researchers. The research proceeds according to the following case setting:

\subsection{Settings of case study}

This experiment examins how designers can deal with the interior space energy problems, which are harmful to people's health and performance. The experiment was one according to the following setting:

\subsubsection{Center Selection}

The ADHD Treatment Centre (HDC). Site analysis according to latitude and longitude was taken from Google earth.

- Latitude : $244554.00 \mathrm{~N}$

- Longitude : :464355.85 S

- Elev. $\quad: 636 \mathrm{~m}$

\subsubsection{Class Selection}

The team chose 4 groups with total 16 selected children at each stage from different environments, as sample from the community (Figures $1 \& 2$ ). 
Figure (1) Location of classes in the building

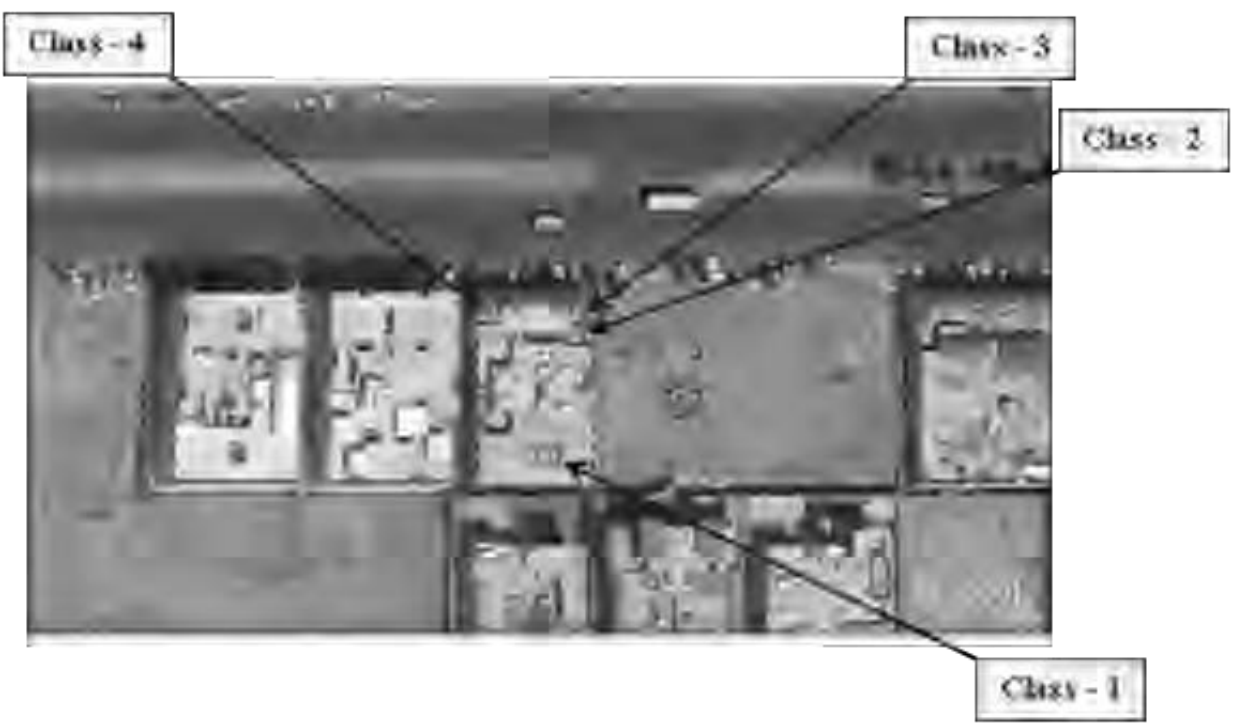

Figure (2) Floor plans of classes

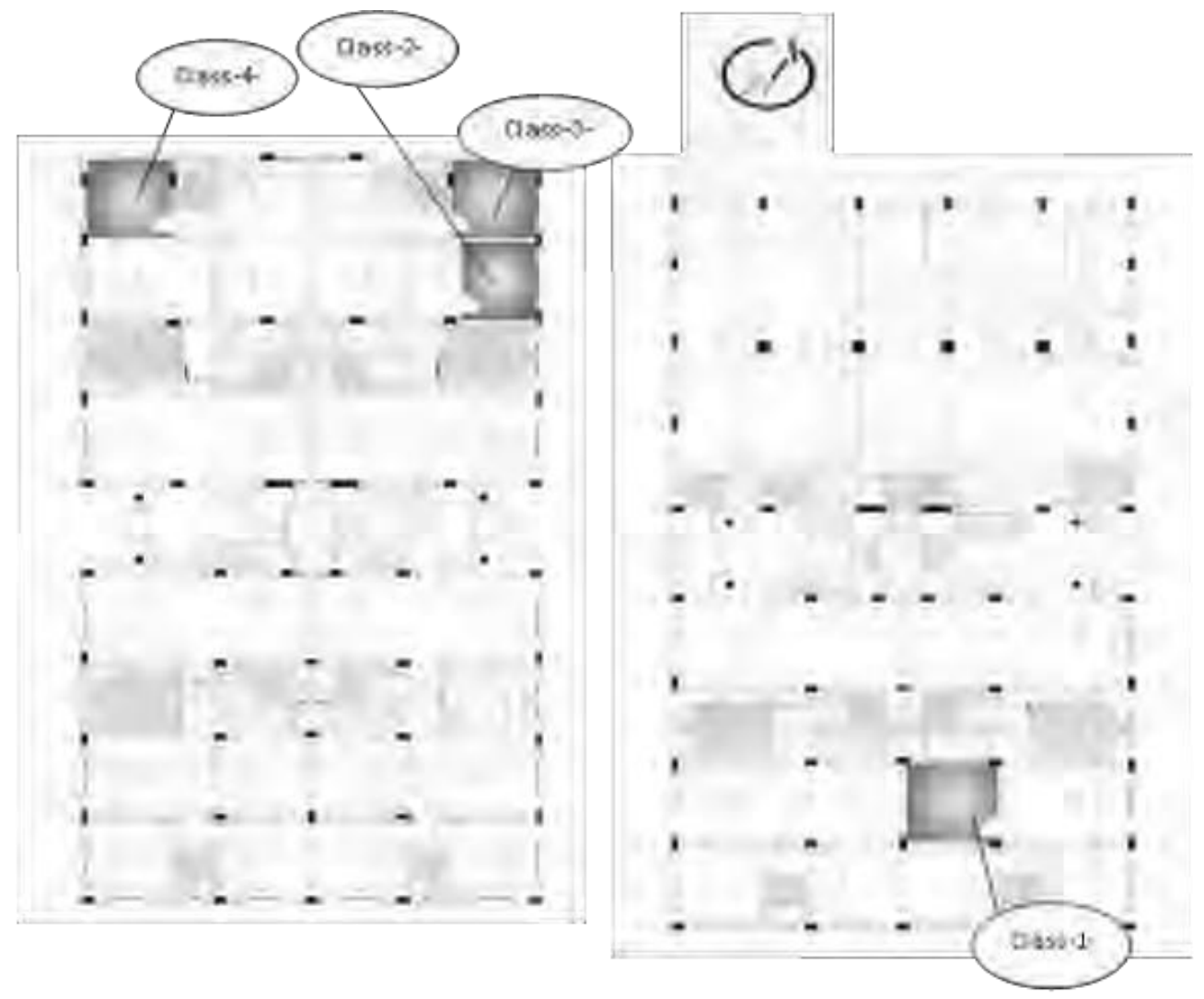

\subsubsection{Student Selection}

The average age between students is from 6-8 years old boys and girls, and the number of students has been chosen for the experiment with the help of one specialist and one assistant. Those children with normal "IQ" 90 and above, all suffer from ADHD and have difficulty in academic achievement. Other causes of learning disability (LD). Those Children have been taken as a sample from the population of the society to work on as research sample. 


\subsubsection{The Medical Crew selection}

The center has specialists who are trained to do psychological tests and result interpretation.

\subsubsection{Time limitation}

Limited time will be 3 months in each stage.

\subsubsection{Tools}

A varity of tools used in this experiment as follows:

\section{Energy Detecting and Balancing Tools and Equipment}

- Tools that will be used to detect the interior space energy are: IK (IKUP) Pendulum, Convective (Virtual Cone Pendulum).

- Tools that will be used to correct the interior space energy are: Dial (for balancing energy coming from doors and windows), and sample collector, in addition to BG cubes (Home Kit), and shapes, and colors

- Tools that will be used to evaluate the interior space energy impact are: Play Attention software program, Checklists, and assessment reports.

- Tools that will be used to analyze and compare the interior space energy results are: excel and SPSS programs

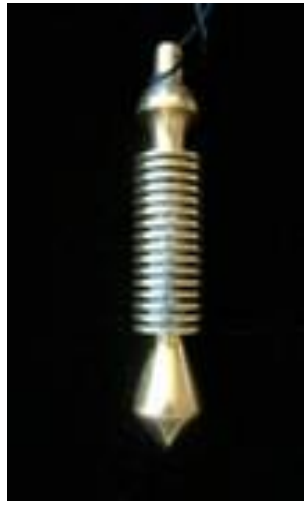

The (IK) Pendulum

Figure (3) Energy Detecting and Balancing Tools

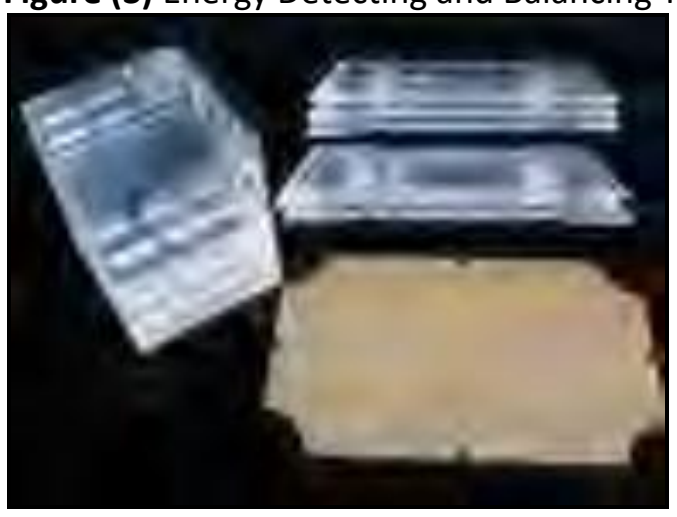

BG Tools (Home Kit) Source: www.biogeometry.com

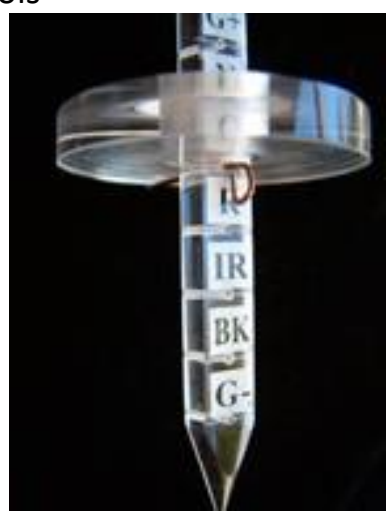

Virtual Cone Pendulum

\section{Play Attention Program:}

The child interns game only with mental focus without touching any button or device to increase his attention and improve his memory and attention.

Figure (4) Play attention: Exam for a student
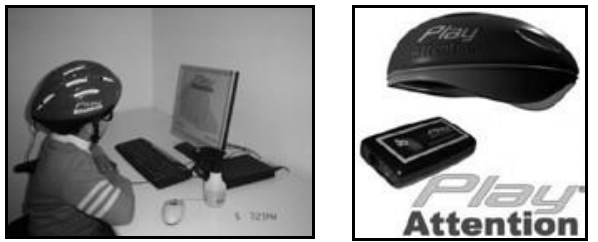

Source: www.ADHD.com 


\section{Colors:}

Colors have been chosen according to a study on the best colors that suites this case.

- Yellow: It stimulates mental ability, focus and a sense of separation and concentration.

- Purple (indigo): This color is the "Chakra" crown of the head, top of higher mind, and the color of dignity, honor, self-esteem the treatment of mental and neurological disorders. It is also a tonic for memory, thinking and cures intestinal disorders and cures breathing disorders.

\subsection{Design Stages}

The Applied study was held in three stages in order to explore the problem, and the role of the researcher was to achieve in order the final results, which have been taken through the experiments stages.

\subsubsection{Stage 1: The Base Case}

The main purpose of the experiment is to use this base case to compare with the other 2 stages results. The whole data about the experiment steps will be given through the following schedule.

Table (1) Base case experiment steps

\begin{tabular}{|c|c|c|c|c|}
\hline Experiment Steps & $\begin{array}{c}\text { Time } \\
\text { Duration }\end{array}$ & Methodology & Evaluation Tools & Results \\
\hline $\begin{array}{l}\text { 1- Selecting the } \\
\text { sample rooms' } \\
\text { allocations } \\
\text { according to the } \\
\text { criteria } \\
\text { mentioned in the } \\
\text { text before. }\end{array}$ & $\begin{array}{l}\text { From: } \\
\text { Jan. -2011 }\end{array}$ & $\begin{array}{l}\text { - Select the classes' } \\
\text { location close to or } \\
\text { above each other, to } \\
\text { have the best results } \\
\text { for the BG Shapes and } \\
\text { Cubes. } \\
\text { - Drew Architectural } \\
\text { Drawings }\end{array}$ & & $\begin{array}{l}\text { (4) } \\
\text { Classes } \\
\text { (3) in the } \\
\text { First floor, } \\
\text { and (1) on } \\
\text { the } \\
\text { ground } \\
\text { floor. }\end{array}$ \\
\hline $\begin{array}{l}\text { 2- Keep all Interior } \\
\text { Spaces as is with } \\
\text { no editing or } \\
\text { changing. }\end{array}$ & $\begin{array}{l}\text { From: } \\
\text { Jan. -2011 } \\
\text { to: } \\
\text { March.2011 }\end{array}$ & $\begin{array}{l}\text { Taking the photos for } \\
\text { classes with no } \\
\text { changing or editing. }\end{array}$ & & \\
\hline $\begin{array}{l}\text { 3- Receiving all the } \\
\text { by weekly } \\
\text { evaluation } \\
\text { reports from the } \\
\text { Centre. }\end{array}$ & $\begin{array}{l}\text { From: } \\
\text { Jan. -2011 } \\
\text { to: } \\
\text { March-2011 }\end{array}$ & $\begin{array}{l}\text { 1- Select (16) Child } \\
\text { diagnosed with } \\
\text { ADHD their ages } \\
\text { between 6-9 with } \\
\text { IQ } 90 \text { and above } \\
\text { 2- Record physicians' } \\
\text { reports and } \\
\text { students' } \\
\text { progress. }\end{array}$ & $\begin{array}{l}\text { 1-Checklists (Doctors, } \\
\text { Teachers, Parents) } \\
\text { 2-Software Programs } \\
\text { (Play Attention) } \\
\text { 3-Reports (Doctors, } \\
\text { Teachers, Parents) }\end{array}$ & $\begin{array}{l}\text { Document } \\
\text { all } \\
\text { students' } \\
\text { progress } \\
\text { results }\end{array}$ \\
\hline $\begin{array}{l}\text { 4- Receiving Final } \\
\text { Evaluation } \\
\text { Reports }\end{array}$ & March-2011 & $\begin{array}{l}\text { 1- Analyze the data and } \\
\text { Sorting it. } \\
\text { 2- Using Excel to } \\
\text { compare progress. } \\
\text { 3- Use the SPSS at the } \\
\text { end of the stages. }\end{array}$ & & \\
\hline
\end{tabular}




\subsubsection{Stage 2: Impact of Colors, BG Cubes and Shapes on Students}

Table (2) Stage 2 experiment steps

\begin{tabular}{|c|c|c|c|c|}
\hline $\begin{array}{c}\text { Experiment } \\
\text { Steps }\end{array}$ & $\begin{array}{c}\text { Time } \\
\text { Duration }\end{array}$ & Methodology & Evaluation Tools & Results \\
\hline $\begin{array}{l}\text { 1- Examine } \\
\text { Interior space } \\
\text { Energy: } \\
\text { Test the } \\
\text { Interior space } \\
\text { Energy (done } \\
\text { by the } \\
\text { researcher). } \\
\text { Fig. } 8\end{array}$ & April-2011 & $\begin{array}{l}\text { 1- Study the site plans } \\
\text { (Google Earth). } \\
\text { 2- Detect the Interior } \\
\text { Grid Lines and interior } \\
\text { space energy in the } \\
\text { whole building } \\
\text { especially the } \\
\text { selected classes (site } \\
\text { visit). } \\
\text { 3- Draw the Grid Lines } \\
\text { on the Architectural } \\
\text { Floor plan. }\end{array}$ & $\begin{array}{l}\text { 1- Special } \\
\text { Pendulums (IK), } \\
\text { Virtual Cone. } \\
\text { 2- Building's floor } \\
\text { plan sketches }\end{array}$ & $\begin{array}{l}\text { Hartman grid } \\
\text { lines became } \\
\text { clear (negative). }\end{array}$ \\
\hline $\begin{array}{l}\text { 2- Selecting } \\
\text { Colors: } \\
\text { 2-1 Testing } \\
\text { and selecting } \\
\text { the best colors } \\
\text { suit this case. } \\
\text { 2-2 Apply and } \\
\text { test The colors } \\
\text { impact on } \\
\text { students } \\
\text { Fig. } 6\end{array}$ & April-2011 & $\begin{array}{l}\text { 1- Select the best } \\
\text { colors to apply to the } \\
\text { selected classes, } \\
\text { regarding the } \\
\text { resonance of the } \\
\text { colors that relate to } \\
\text { the chakras, and } \\
\text { monitor the } \\
\text { progress. } \\
2 \text { Select wall paint } \\
\text { colors yellow and } \\
\text { purple (indigo). }\end{array}$ & $\begin{array}{l}\text { 1- Special } \\
\text { Pendulums. } \\
\text { 2- Color studies } \\
\text { mentioned in } \\
\text { chapter two. } \\
\text { 3- Medical Reports. } \\
\text { 4- Play attention } \\
\text { Software } \\
\text { 5- Checklists } \\
\text { (Doctors, teachers, } \\
\text { Parents) }\end{array}$ & $\begin{array}{l}\text { Students' } \\
\text { behavior started } \\
\text { to change in } \\
\text { classes }\end{array}$ \\
\hline $\begin{array}{l}\text { 3- Adding the } \\
\text { BG Cubes and } \\
\text { shapes: Using } \\
\text { the } 3 \text { BG cubes } \\
\text { and stripes in } \\
\text { the selected } \\
\text { classes (done } \\
\text { by the } \\
\text { researcher), } \\
\text { Fig. } 7\end{array}$ & April & $\begin{array}{l}\text { To balance the energy } \\
\text { in the interior space. } \\
\text { 1- Select the no. of BG } \\
\text { cubes to cover the } \\
\text { whole area. } \\
\text { 2- Daily recharge for the } \\
\text { cubes on it is tray } 5 \\
\text { min. } \\
\text { 3- Washing cubes twice } \\
\text { a week. } \\
\text { 4- Recieve Physicians } \\
\text { report and monitor } \\
\text { the progress. }\end{array}$ & $\begin{array}{l}\text { 1- Checklists } \\
\text { (Physicians, } \\
\text { teachers, } \\
\text { Parents) } \\
\text { 2- Software } \\
\text { Programs } \\
\text { (play attention) } \\
\text { 3- Reports } \\
\text { (Physicians, } \\
\text { teachers, Parents) } \\
\text { 4- Pendulums. }\end{array}$ & $\begin{array}{l}\text { Students' } \\
\text { behavior and } \\
\text { concentration } \\
\text { continued in } \\
\text { changing classes } \\
\text { to the best. }\end{array}$ \\
\hline $\begin{array}{l}\text { 4- Dealing with } \\
\text { EMF in the } \\
\text { interior space } \\
\text { Fig. } 6\end{array}$ & April-2011 & $\begin{array}{l}\text { Strips are placed on } \\
\text { the electricity, } \\
\text { appliances, lighting } \\
\text { wires, on any electrical } \\
\text { device wire and in the } \\
\text { main electric box. }\end{array}$ & $\begin{array}{l}\text { Same previous } \\
\text { Tools }\end{array}$ & $\begin{array}{l}\text { Students } \\
\text { continued on } \\
\text { getting better } \\
\text { and looks comfy } \\
\text { and relax }\end{array}$ \\
\hline $\begin{array}{l}\text { 5- Receiving all } \\
\text { by weekly } \\
\text { reports and } \\
\text { all final } \\
\text { evaluation } \\
\text { reports }\end{array}$ & $\begin{array}{l}\text { From: April- } \\
2011 \text { To: } \\
\text { June-2011 }\end{array}$ & $\begin{array}{l}\text { 1-Analyze and Sort the } \\
\text { given data. } \\
\text { 2-Using Excel program } \\
\text { to compare results. } \\
\text { 3-Using SPSS to } \\
\text { compare results }\end{array}$ & $\begin{array}{l}\text { Same previous } \\
\text { Tools }\end{array}$ & $\begin{array}{l}\text { Document } \\
\text { students' } \\
\text { progress and } \\
\text { compare results } \\
\text { to evaluate their } \\
\text { performance }\end{array}$ \\
\hline
\end{tabular}

EMF: Electro Magnetic Fields -BG: Bio-Geometry 
Figure (5) Choosing and applying the color

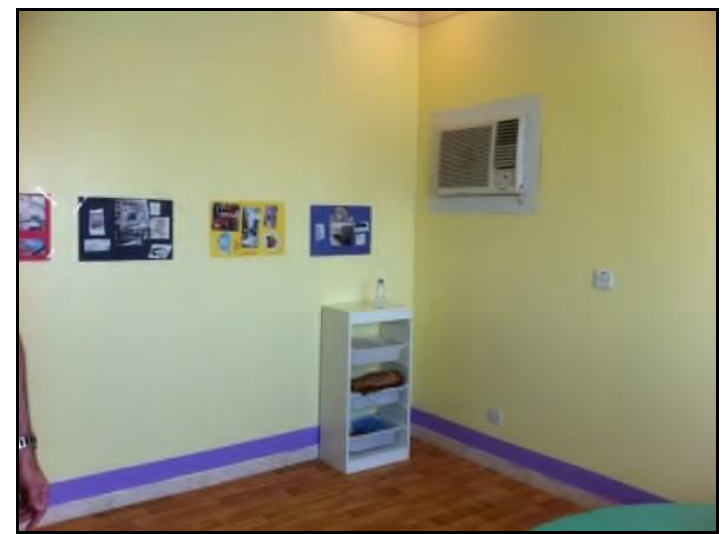

Figure (7) Class-1-after changing the colors in the classes

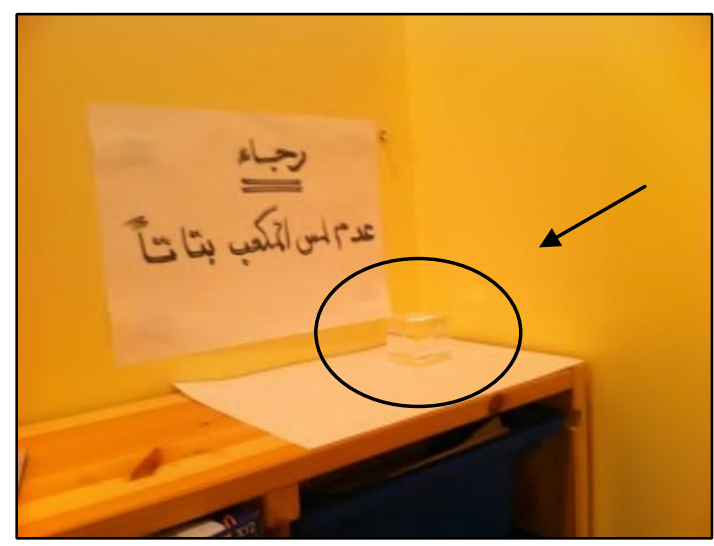

Figure (6) EMF is corrected using BG strips on wires

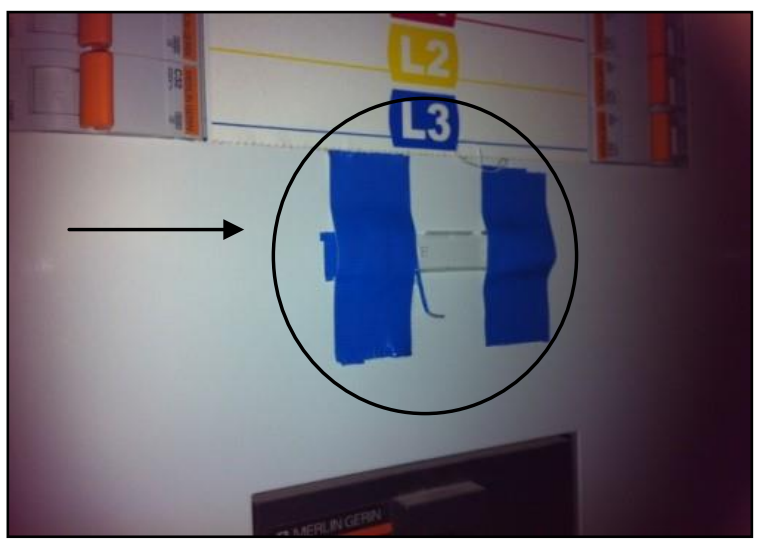

Figure (8) First Floor Plan 


\subsubsection{Stage 3: Interior Architectural spaces and window designs to balance energy}

Table (3) Stage 3 experiment steps

\begin{tabular}{|c|c|c|c|c|}
\hline $\begin{array}{c}\text { Experiment } \\
\text { Steps }\end{array}$ & $\begin{array}{c}\text { Time } \\
\text { Duration }\end{array}$ & Methodology & Evaluation Tools & Results \\
\hline $\begin{array}{l}\text { Balancing the } \\
\text { Interior Space } \\
\text { Energy: } \\
\text { Windows } \\
\text { Designs (done } \\
\text { by the } \\
\text { researcher). }\end{array}$ & Oct-2011 & 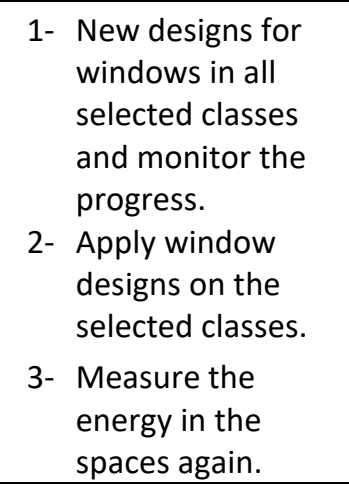 & $\begin{array}{ll}\text { 1- } & \text { Special } \\
& \text { Pendulums } \\
\text { 2- } & \text { Medical } \\
& \text { Reports } \\
\text { 3- } & \text { Play Attention } \\
& \text { Software } \\
\text { 4- } & \text { Checklists }\end{array}$ & $\begin{array}{l}\text { The energy coming } \\
\text { from windows has } \\
\text { been changed from } \\
\text { negative to positive, } \\
\text { and energy in the } \\
\text { space was balanced. } \\
\text { Students' behavior } \\
\text { changed in classes. }\end{array}$ \\
\hline $\begin{array}{l}\text { Doors energy } \\
\text { balance (done } \\
\text { by the } \\
\text { researcher). } \\
\text { Collecting } \\
\text { material } \\
\text { samples to } \\
\text { balance the } \\
\text { interior space } \\
\text { energy }\end{array}$ & Oct. -2011 & 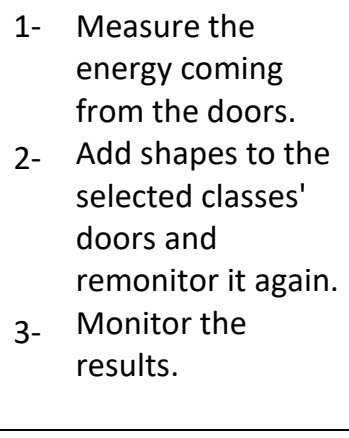 & $\begin{array}{l}\text { Same previous } \\
\text { Tools }\end{array}$ & $\begin{array}{l}\text { The energy coming } \\
\text { from doors has } \\
\text { been changed from } \\
\text { negative to positive, } \\
\text { and energy was } \\
\text { balanced. Students' } \\
\text { behavior and } \\
\text { concentration } \\
\text { continued in } \\
\text { changing in classes. }\end{array}$ \\
\hline $\begin{array}{l}\text { Receiving all by } \\
\text { weekly reports } \\
\text { and all Final } \\
\text { Evaluation } \\
\text { Reports }\end{array}$ & Jan-2012 & $\begin{array}{l}\text { 1- Analyze and sort } \\
\text { the data Using } \\
\text { 2- Excel to } \\
\text { compare } \\
\text { results. }\end{array}$ & $\begin{array}{l}\text { Same previous } \\
\text { tools }\end{array}$ & $\begin{array}{l}\text { Document all } \\
\text { students' progress } \\
\text { and compare results } \\
\text { to evaluate their } \\
\text { performance }\end{array}$ \\
\hline
\end{tabular}

Figure (9) Using dial to balance the energy

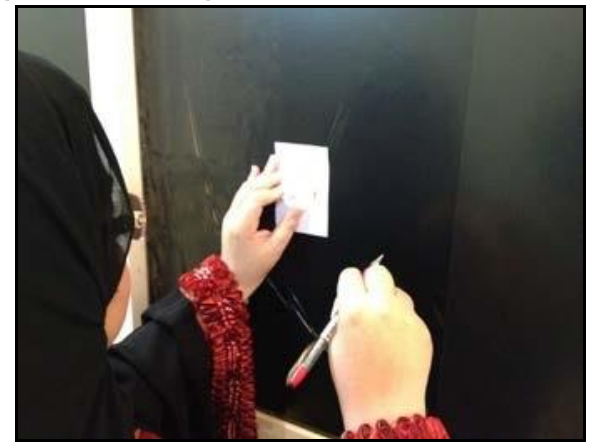

Figure (11) Collecting samples

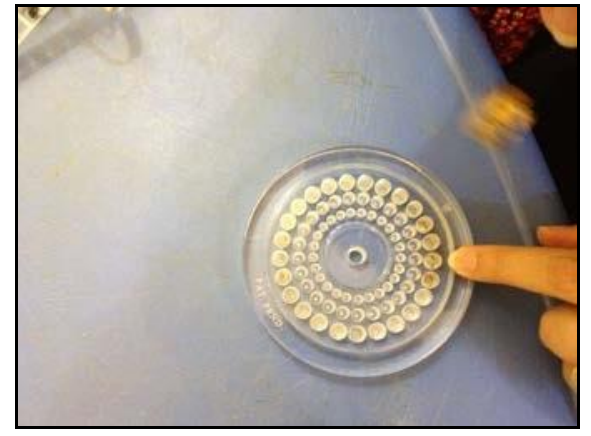

Figure (10) Applying another window design

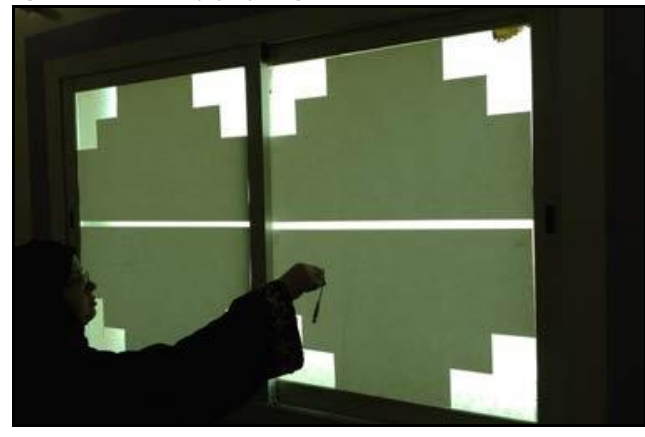

Figure (12) Balance the doors energy

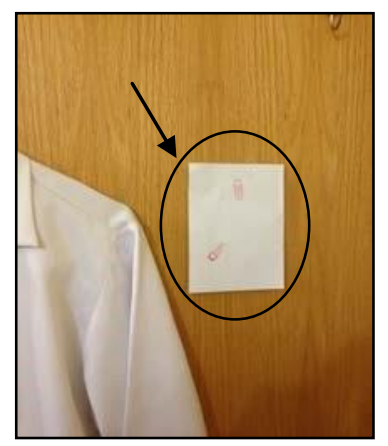




\subsubsection{Pretest}

Table (4) Results from the Assessment Diagnoses Reports in Stage 1 (Sample for all stages)

\begin{tabular}{|c|c|c|c|c|c|c|c|c|c|c|c|}
\hline No & 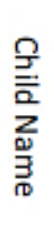 & 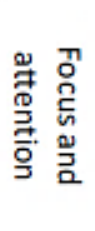 & 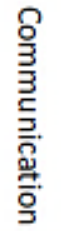 & 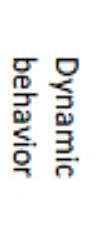 & 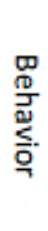 & 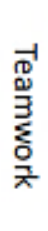 & 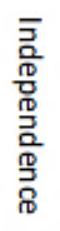 & $\begin{array}{l}\text { I } \\
\text { 方 } \\
\text { D } \\
\frac{D}{2}\end{array}$ & $\begin{array}{l}\frac{\varkappa}{0} \\
\frac{O}{0} \\
\frac{n}{\hat{n}} \\
\overline{\bar{n}}\end{array}$ & 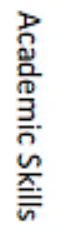 & 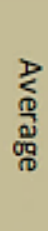 \\
\hline
\end{tabular}

Stage 1: Group One -Supervisor: Hitaf- Diagnoses"Jan-2011"

\begin{tabular}{cccccccccccc}
\hline 1 & Motaz & 3 & 2.75 & 2.8 & 2.7 & 3 & 2 & 2.9 & 2.25 & 2.6 & 2.85 \\
\hline 2 & Loai & 3 & 3 & 3 & 2.25 & 2.8 & 2.25 & 3 & 2.25 & 3 & 2.81 \\
\hline 3 & Judi & 2.8 & 3 & 2.75 & 3 & 2.7 & 3 & 3 & 2.25 & 2.85 & 2.85 \\
\hline 4 & $\begin{array}{c}\text { Abdul } \\
\text { Rahman }\end{array}$ & 3 & 2.85 & 3 & 3 & 3 & 2.5 & 3 & 2.5 & 3 & 2.97 \\
\hline
\end{tabular}

Stage 1: Group Two -Supervisor: Mona-Diagnoses" Jan-2011"

\begin{tabular}{cccccccccccc}
\hline 1 & Meshal & 3 & 3 & 3 & 2.85 & 2.5 & 2.5 & 2.75 & 2.5 & 3 & 2.87 \\
\hline 2 & Abdulla & 2.7 & 3 & 2.1 & 3 & 3 & 2 & 2 & 3 & 2 & 2.76 \\
\hline 3 & Bandar & 3 & 2.75 & 3 & 3 & 2.8 & 2 & 3 & 3 & 3 & 2.91 \\
\hline 4 & Maria & 2.9 & 3 & 2 & 2.75 & 3 & 3 & 2 & 2.25 & 3 & 2.73 \\
\hline
\end{tabular}

\begin{tabular}{|c|c|c|c|c|c|c|c|c|c|c|c|}
\hline \multicolumn{12}{|c|}{ Stage 1: Group Three -Supervisor: Hanouf- Diagnoses"Jan-2011" } \\
\hline 1 & Daoud & 3 & 2.9 & 3 & 2.25 & 2.5 & 2.5 & 2.75 & 2.5 & 2.85 & 2.73 \\
\hline 2 & Ahmad & 3 & 2.6 & 2.4 & 3 & 2 & 2 & 3 & 2.5 & 2.5 & 2.60 \\
\hline 3 & Rayyan & 3 & 3 & 2.1 & 3 & 2.75 & 2.9 & 2.85 & 2 & 2.8 & 2.77 \\
\hline 4 & Turki & 3 & 2.7 & 3 & 2.95 & 2.86 & 2 & 2.8 & 2.5 & 2.8 & 2.90 \\
\hline
\end{tabular}

Stage 1: Group Four -Supervisor: Amira-Diagnoses-"Jan-2011"

\begin{tabular}{llcccccccccc}
\hline 1 & Lama & 2.8 & 3 & 3 & 2.5 & 3 & 2 & 3 & 2.5 & 2.75 & 2.86 \\
\hline 2 & Sara & 2.4 & 2.85 & 2.75 & 3 & 3 & 2.85 & 3 & 2.5 & 2.85 & 2.80 \\
\hline 3 & Ahmad & 3 & 3 & 3 & 2.9 & 2.5 & 2.25 & 2.95 & 3 & 2.5 & 2.88 \\
\hline 4 & Salem & 3 & 2.8 & 3 & 3 & 3 & 2.8 & 2.75 & 2.25 & 3 & 2.96 \\
\hline \multicolumn{2}{l}{ Criteria: } & 1: Improved & \multicolumn{2}{l}{ 2: Do the action with help } & \multicolumn{3}{c}{ 3: Needs much help } \\
\hline
\end{tabular}

Table (5) The experiment starts assessment diagnoses report -Stage 2

\begin{tabular}{|c|c|c|c|c|c|c|c|c|c|c|c|}
\hline No & 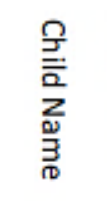 & 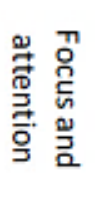 & 올 & 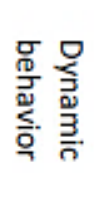 & 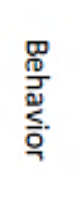 & 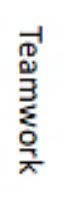 & 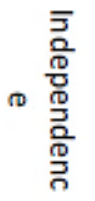 & 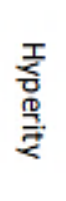 & 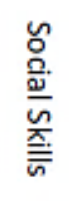 & 只 & 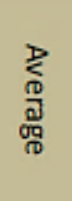 \\
\hline \multicolumn{12}{|c|}{ Stage 2: Group One -Supervisor: Hitaf- Diagnoses"April-2011" } \\
\hline 1 & Gaidaa & 2.9 & 2.8 & 2.7 & 3 & 3 & 2 & 2.9 & 2.25 & 2.7 & 2.88 \\
\hline 2 & Abdulla & 3 & 3 & 3 & 2.25 & 2.8 & 2.25 & 3 & 2.25 & 3 & 2.81 \\
\hline 3 & $\begin{array}{l}\text { Moha } \\
\text { mmad }\end{array}$ & 2.8 & 3 & 2.75 & 3 & 3 & 3 & 3 & 2.25 & 2.85 & 2.9 \\
\hline 4 & Nawaf & 2.95 & 2.85 & 2.7 & 3 & 3 & 2.5 & 3 & 2.5 & 3 & 2.9 \\
\hline
\end{tabular}

Stage 2: Group Two -Supervisor: Mona- Diagnoses" ${ }^{\text {April-2011" }}$

\begin{tabular}{cccccccccccc}
\hline 1 & Meshal & 3 & 3 & 3 & 2.85 & 2.5 & 2.5 & 2.75 & 2.5 & 3 & 2.87 \\
\hline 2 & Abdulla & 2.7 & 3 & 2.1 & 3 & 3 & 2 & 2 & 3 & 2 & 2.76 \\
\hline 3 & Bandar & 3 & 2.75 & 3 & 3 & 2.8 & 2 & 3 & 3 & 3 & 2.91 \\
\hline 4 & Maria & 2.9 & 3 & 2 & 2.75 & 3 & 3 & 2 & 2.25 & 3 & 2.73 \\
\hline
\end{tabular}




\begin{tabular}{|c|c|c|c|c|c|c|c|c|c|c|c|}
\hline No & 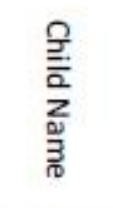 & 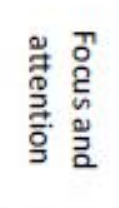 & 옹 & 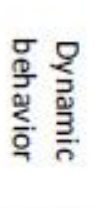 & 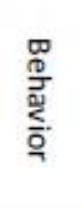 & 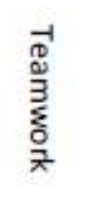 & 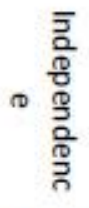 & 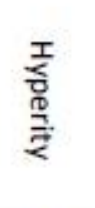 & $\begin{array}{l}\frac{\varkappa}{0} \\
\frac{\frac{2}{0}}{n} \\
\frac{\bar{N}}{\bar{n}}\end{array}$ & 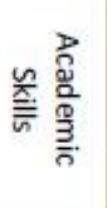 & 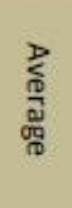 \\
\hline \multicolumn{12}{|c|}{ Stage 2: Group Three -Supervisor: Hanouf-Diagnoses"April-2011" } \\
\hline 1 & Sara & 2.7 & 2.9 & 2.5 & 2.25 & 2.5 & 2.5 & 2.75 & 2.5 & 2 & 2.75 \\
\hline 2 & $\begin{array}{c}\text { Abdul- } \\
\text { Aziz }\end{array}$ & 3 & 2.6 & 2.4 & 3 & 2 & 2 & 3 & 2.5 & 2.5 & 2.60 \\
\hline 3 & Merdas & 3 & 3 & 2.1 & 3 & 2.75 & 2.9 & 2.85 & 2 & 2.8 & 2.80 \\
\hline 4 & Turki & 3 & 2.7 & 3 & 2.95 & 2.86 & 2 & 2.8 & 2.5 & 2.8 & 2.90 \\
\hline \multicolumn{12}{|c|}{ Stage 2: Group Four -Supervisor: Amira-Diagnoses -"April-2011" } \\
\hline 1 & Soliman & 2.8 & 2.8 & 2.6 & 2.5 & 2.85 & 2 & 3 & 2.5 & 2.7 & 2.70 \\
\hline 2 & Faisal & 2.4 & 2.85 & 2.75 & 3 & 3 & 2.85 & 3 & 2.5 & 2.85 & 2.80 \\
\hline 3 & Turki & 3 & 3 & 3 & 2.9 & 2.5 & 2.25 & 2.95 & 3 & 2.5 & 2.88 \\
\hline 4 & Ibrahim & 3 & 2.8 & 3 & 2.7 & 3 & 2.8 & 2.75 & 2.25 & 2.7 & 2.90 \\
\hline \multicolumn{2}{|c|}{ Criteria: } & \multicolumn{2}{|c|}{ 1: Improved } & \multicolumn{4}{|c|}{ 2: Do the action with help } & \multicolumn{3}{|c|}{ 3: Needs much help } & \\
\hline
\end{tabular}

Table (6) The experimental results from diagnoses reports - Stage 3

\begin{tabular}{|c|c|c|c|c|c|c|c|c|c|c|c|}
\hline No & 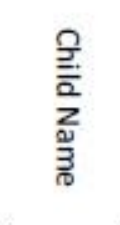 & 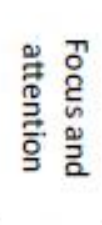 & $=\frac{\stackrel{2}{O}}{3}$ & 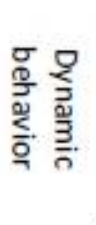 & 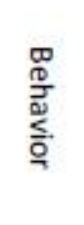 & $\begin{array}{l}-1 \\
\frac{1}{3} \\
\frac{2}{3} \\
\frac{1}{\pi} \\
\frac{7}{\pi}\end{array}$ & 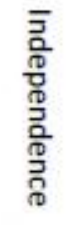 & 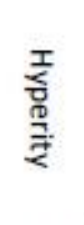 & 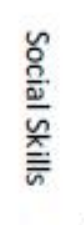 & 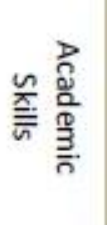 & 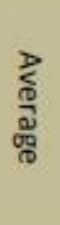 \\
\hline \multicolumn{12}{|c|}{ Stage 3: Group One -Supervisor: Hitaf- Diagnoses" Oct. -2011" } \\
\hline 1 & Fahad & 2.9 & 2.2 & 2.75 & 2 & 2 & 2 & 2.5 & 2 & 2.4 & 2.4 \\
\hline 2 & Daoud & 3 & 2 & 2 & 3 & 2 & 3 & 2.4 & 3 & 3 & 2.4 \\
\hline 3 & Ahmad & 2.6 & 2 & 2.9 & 2.96 & 2.7 & 3 & 2.6 & 3 & 2.4 & 2.6 \\
\hline 4 & Rayyan & 3 & 2.3 & 2.3 & 3 & 2 & 2 & 2.7 & 2.9 & 3 & 2.5 \\
\hline \multicolumn{12}{|c|}{ Stage 3: Group Two -Supervisor: Mona- Diagnoses" "Oct.-2011" } \\
\hline 1 & $\begin{array}{c}\text { Abdul- } \\
\text { Aziz }\end{array}$ & 2.8 & 3 & 2.7 & 3 & 2.7 & 2.8 & 3 & 2.8 & 2.9 & 2.9 \\
\hline 2 & Khaled & 2.95 & 2.85 & 2.95 & 2.9 & 3 & 3 & 3 & 2.7 & 2.9 & 2.9 \\
\hline 3 & Naeif & 3 & 3 & 3 & 2.8 & 3 & 2.25 & 2.95 & 3 & 2.5 & 3.0 \\
\hline 4 & Omair & 3 & 2.6 & 2.8 & 3 & 2.7 & 2.8 & 2.8 & 2.5 & 3 & 2.8 \\
\hline \multicolumn{12}{|c|}{ Stage 3: Group Three -Supervisor: Hanouf- Diagnoses "Oct.-2011" } \\
\hline 1 & Abdullah & 2.6 & 2.8 & 2.83 & 3 & 3 & 3 & 2.5 & 2.9 & 2.9 & 2.8 \\
\hline 2 & Naser & 2.5 & 2.8 & 2.85 & 3 & 2.9 & 2.7 & 2.5 & 2.6 & 3 & 2.8 \\
\hline 3 & Gazal & 3 & 2.4 & 2.9 & 2.96 & 2.2 & 2 & 2.71 & 2.95 & 3 & 2.7 \\
\hline 4 & Naief & 2.9 & 3 & 2.85 & 2.3 & 3 & 2.9 & 3 & 3 & 2.2 & 2.8 \\
\hline \multicolumn{12}{|c|}{ Stage 3: Group Four -Supervisor: Amira- Diagnoses"Oct.-2011" } \\
\hline 1 & $\begin{array}{c}\text { Solima } \\
\text { n }\end{array}$ & 3 & 2.9 & 3 & 2.25 & 3 & 2.5 & 2.5 & 2.5 & 2.85 & 2.8 \\
\hline 2 & Faisal & 2.9 & 2.5 & 2.5 & 2.6 & 2.7 & 2 & 3 & 2.6 & 2.6 & 2.7 \\
\hline 3 & Turki & 3 & 3 & 3 & 3 & 3 & 2.9 & 2.95 & 2 & 3 & 3.0 \\
\hline 4 & Ibrahim & & 2.6 & 3 & 2.7 & 3 & 2 & 3 & 2.5 & 2.8 & 2.9 \\
\hline \multicolumn{4}{|c|}{ 1: Improved } & \multicolumn{4}{|c|}{ 2: Do the action with help } & \multicolumn{4}{|c|}{ 3: Needs much help } \\
\hline
\end{tabular}


3.2.5 Post Test (After the experiment 3-months)

Table (7) Results for Assessment final report - Stage 1

\begin{tabular}{|c|c|c|c|c|c|c|c|c|c|c|c|}
\hline No & 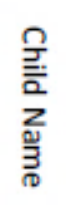 & 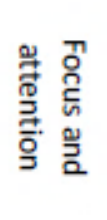 & 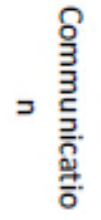 & 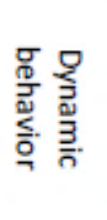 & 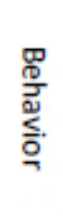 & 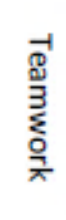 & 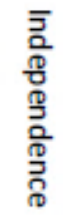 & 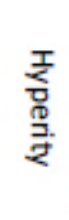 & $\begin{array}{l}\frac{n}{0} \\
\frac{\frac{C}{0}}{0} \\
\frac{n}{\bar{n}} \\
\overline{\bar{n}}\end{array}$ & 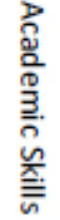 & 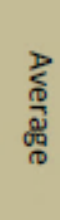 \\
\hline
\end{tabular}

Stage 1: Group One -Supervisor: Hitaf- Final Report "March-2011"

\begin{tabular}{cccccccccccc}
\hline 1 & Motaz & 2.8 & 2.2 & 2 & 2.1 & 2 & 2.2 & 2.1 & 2.7 & 1.4 & 2.2 \\
\hline 2 & Loai & 2.7 & 2.2 & 2 & 3 & 2 & 2.3 & 2.4 & 3 & 3 & 2.4 \\
\hline 3 & Judi & 2 & 2 & 2 & 3 & 2 & 1 & 2 & 3 & 2.4 & 2.2 \\
\hline 4 & $\begin{array}{c}\text { Abdul } \\
\text { Rahman }\end{array}$ & 1.9 & 2.2 & 1.6 & 3 & 2 & 2 & 1.95 & 2.9 & 3 & 2.1 \\
\hline
\end{tabular}

Stage 1: Group Two -Supervisor: Mona - Final Report "March-2011"

\begin{tabular}{cccccccccccc}
\hline 1 & Meshal & 2.5 & 2.4 & 2.4 & 2 & 2.5 & 2.8 & 2.4 & 2.85 & 2.7 & 2.5 \\
\hline 2 & Abdulla & 2.2 & 2.2 & 2.8 & 2 & 1.4 & 2 & 2.1 & 3 & 2.2 & 2.2 \\
\hline 3 & Bandar & 2 & 2.5 & 2 & 2.2 & 2 & 2 & 1.4 & 3 & 3 & 2.2 \\
\hline 4 & Maria & 2.6 & 2.2 & 2.1 & 2.3 & 2 & 2.2 & 2 & 2.25 & 2.3 & 2.2 \\
\hline
\end{tabular}

Stage 1: Group Three -Supervisor: Hanouf - Final Report "March-2011"

\begin{tabular}{llcccccccccc}
\hline 1 & Daoud & 2 & 2 & 2 & 2 & 3 & 2 & 2.1 & 2.5 & 2.85 & 2.2 \\
\hline 2 & Ahmad & 1.75 & 1.96 & 1.85 & 1.85 & 2.4 & 1.8 & 1.6 & 2.5 & 2.3 & 2.0 \\
\hline 3 & Rayyan & 2 & 3 & 2 & 2 & 2 & 2 & 2.1 & 2 & 2.8 & 2.2 \\
\hline 4 & Turki & 1.9 & 1.8 & 2 & 1.9 & 1.8 & 2.2 & 2 & 2.6 & 2.7 & 2.2 \\
\hline
\end{tabular}

Stage 1: Group Four -Supervisor: Amira- Final Report "March-2011"

\begin{tabular}{llcccccccccc}
\hline 1 & Lama & 2 & 2 & 2 & 1.7 & 2.2 & 2 & 1.97 & 2.5 & 2.8 & 2.1 \\
\hline 2 & Sara & 1 & 1.8 & 2 & 1.8 & 2 & 2.7 & 1.8 & 2.5 & 2.6 & 2.0 \\
\hline 3 & Ahmad & 2 & 1.5 & 1.7 & 2 & 2 & 2.25 & 1.85 & 3 & 2.6 & 2.1 \\
\hline 4 & Salem & 1.8 & 2.3 & 2.2 & 2.4 & 2 & 2.8 & 2.14 & 2.6 & 3 & 2.4 \\
\hline \multicolumn{1}{l}{ Criteria: } & 1: Improved & 2: Do the action with help & \multicolumn{2}{c}{ 3: Needs much help } \\
\hline
\end{tabular}

Table (8) The experiments Results for The Final Report - Stage 2

\begin{tabular}{|c|c|c|c|c|c|c|c|c|c|c|c|}
\hline No & 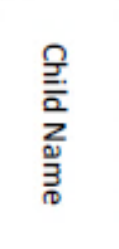 & 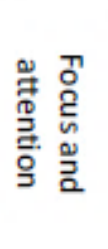 & 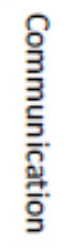 & 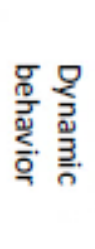 & 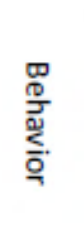 & \begin{tabular}{l}
-1 \\
$\stackrel{0}{0}$ \\
3 \\
\multirow{3}{3}{} \\
$\frac{7}{\pi}$
\end{tabular} & 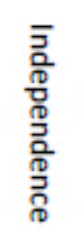 & 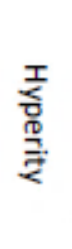 & 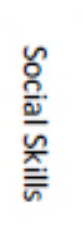 & 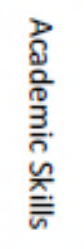 & 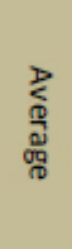 \\
\hline \multicolumn{12}{|c|}{ Stage 2: Group One -Supervisor: Hitaf- Final Report "June-2011" } \\
\hline 1 & Gaidaa & 1.4 & 1.5 & 1.6 & 1.5 & 1.85 & 1.25 & 1.7 & 1.5 & 1.5 & 1.5 \\
\hline 2 & Abdulla & 1.7 & 1.7 & 2 & 1.6 & 1.5 & 1.5 & 1.5 & 2.5 & 2.5 & 1.5 \\
\hline 3 & $\begin{array}{l}\text { Moha } \\
\text { mmad }\end{array}$ & 1.7 & 1 & 1.6 & 1.7 & 1.75 & 2 & 2 & 2 & 3 & 1.6 \\
\hline 4 & $\begin{array}{c}\text { Nawwa } \\
f\end{array}$ & 2 & 1.75 & 1.6 & 1.7 & 1 & 2 & 1.5 & 2.5 & 2.8 & 1.6 \\
\hline \multicolumn{12}{|c|}{ Stage 2: Group Two -Supervisor: Mona - Final Report "June-2011" } \\
\hline 1 & Meshal & 1.85 & 2 & 1.3 & 2.25 & 2 & 2.5 & 2 & 2.5 & 1.75 & 1.88 \\
\hline 2 & Abdulla & 1 & 1.75 & 2 & 2.25 & 1.75 & 1.7 & 1 & 1.25 & 2 & 1.75 \\
\hline 3 & Bandar & 1.5 & 1.7 & 1.3 & 2 & 1.5 & 2.25 & 2 & 1.5 & 2 & 1.6 \\
\hline 4 & Maria & 2.75 & 1.75 & 2 & 1.5 & 1 & 2.5 & 1 & 2.75 & 2 & 1.8 \\
\hline
\end{tabular}




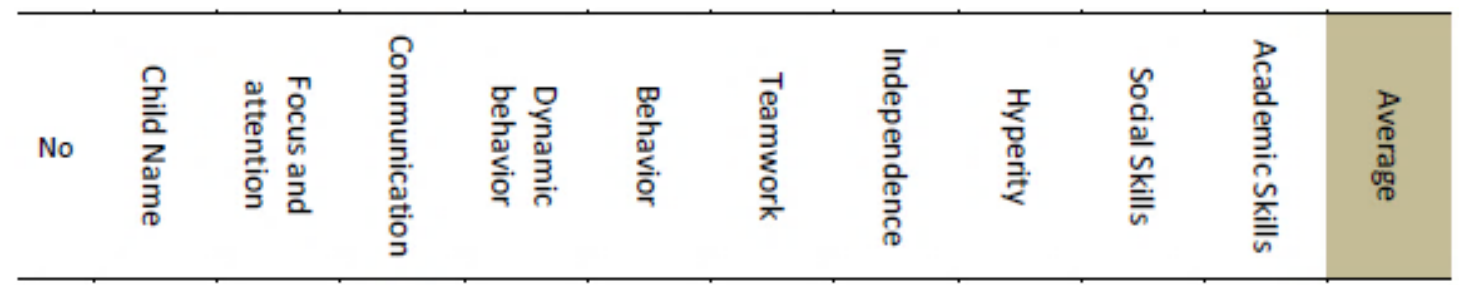

Stage 2: Group Three -Supervisor: Hanouf - Final Report "June-2011"

\begin{tabular}{lccccccccccc}
\hline 1 & Sara & 1.7 & 1.6 & 1.7 & 1.7 & 1.5 & 2.0 & 1.6 & 2.1 & 2.0 & 1.6 \\
\hline 2 & $\begin{array}{c}\text { Abdul- } \\
\text { Aziz }\end{array}$ & 1.7 & 1.6 & 1.7 & 1.8 & 1.5 & 2.0 & 1.6 & 2.1 & 2.2 & 1.7 \\
\hline 3 & Merdas & 1.7 & 1.6 & 1.7 & 1.8 & 1.8 & 2.0 & 1.7 & 2.1 & 2.2 & 1.7 \\
\hline 4 & Turki & 1.9 & 2.4 & 2.4 & 1.0 & 1.0 & 2.3 & 1.5 & 2.3 & 2.3 & 1.7 \\
\hline
\end{tabular}

Stage 2: Group Four -Supervisor: Amira- Final Report "June-2011"

\begin{tabular}{lccccccccccc}
\hline 1 & Soliman & 1.7 & 1.6 & 1.8 & 1.8 & 1.5 & 2 & 2 & 1.7 & 1.5 & 1.7 \\
\hline 2 & Faisal & 1.4 & 1.25 & 2 & 1.25 & 2.25 & 1.5 & 1 & 1.5 & 2 & 1.6 \\
\hline 3 & Turki & 2 & 1 & 1.5 & 2 & 1.5 & 1.5 & 2 & 1.5 & 2 & 1.6 \\
\hline 4 & Ibrahim & 1.7 & 1.5 & 2 & 1.5 & 1.6 & 1.5 & 1.2 & 2 & 2 & 1.7 \\
\hline \multicolumn{2}{l}{ Criteria: } & 1: Improved & \multicolumn{2}{c}{ 2: Do the action with help } & 3: Neds much help \\
\hline
\end{tabular}

Table (9) The experiments final reports - Stage 3

\begin{tabular}{|c|c|c|c|c|c|c|c|c|c|c|c|}
\hline No & 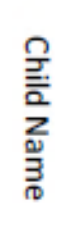 & 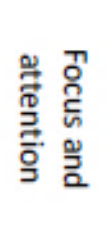 & 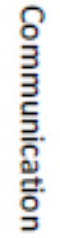 & 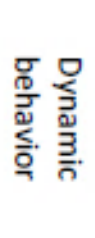 & 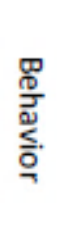 & 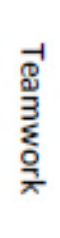 & 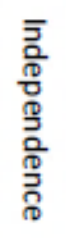 & 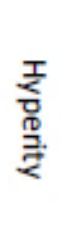 & 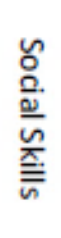 & 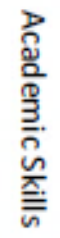 & $\underset{\substack{\mathbb{d} \\
\text { 离 }}}{\stackrel{D}{d}}$ \\
\hline
\end{tabular}

Stage 3: Group One -Supervisor: Hitaf- Final Report "Jan. -2012"

\begin{tabular}{cccccccccccc}
\hline 1 & Fahad & 1.3 & 1.3 & 1.6 & 1.5 & 1.4 & 2 & 1.6 & 1 & 1.4 & 1.5 \\
\hline 2 & Daoud & 1.3 & 1.5 & 1.7 & 1.8 & 1.6 & 1.5 & 1.2 & 1.2 & 1.5 & 1.6 \\
\hline 3 & Ahmad & 1.5 & 1.2 & 1.6 & 2 & 1.9 & 2 & 2 & 1.5 & 2.3 & 1.6 \\
\hline 4 & Ibrahim & 2 & 1.75 & 2 & 1.5 & 1.3 & 2 & 1.4 & 1.8 & 1.7 & 1.7 \\
\hline
\end{tabular}

Stage 3: Group Two -Supervisor: Mona - Final Report "Jan. -2012"

\begin{tabular}{cccccccccccc}
\hline 1 & $\begin{array}{c}\text { Abdul- } \\
\text { Aziz }\end{array}$ & 1.5 & 1.6 & 1.5 & 1.7 & 1.5 & 1.6 & 1.7 & 1.4 & 1.8 & 1.6 \\
\hline 2 & Khaled & 1.6 & 1.6 & 1.4 & 1.5 & 1.4 & 1.3 & 1.5 & 1.4 & 1.3 & 1.5 \\
\hline 3 & Naeif Z & 1.6 & 2 & 1.6 & 2 & 1.6 & 1.7 & 1.7 & 1.4 & 1.8 & 1.8 \\
\hline 4 & Omair & 1.7 & 1.6 & 1.4 & 1.5 & 1.3 & 1.5 & 1.6 & 1.5 & 1.7 & 1.5 \\
\hline \multicolumn{7}{c}{ Stage 3: Group Three -Supervisor: Hanouf - Final Report "Jan. $-2012^{\prime \prime}$} \\
\hline 1 & Abdullah & 1.8 & 1.5 & 1.5 & 1.3 & 2 & 1 & 1.5 & 1.2 & 1.6 & 1.6 \\
\hline 2 & Naser & 1.7 & 1.7 & 1.4 & 1.3 & 1.4 & 2 & 1.9 & 1.4 & 1.6 & 1.5 \\
\hline 3 & Gazal & 1.6 & 1.2 & 1.6 & 1.2 & 1.5 & 2 & 1.6 & 1.2 & 1.5 & 1.4 \\
\hline 4 & Naief M & 1.9 & 1.5 & 2 & 1.3 & 1.4 & 2 & 1.6 & 1.3 & 1.6 & 1.6 \\
\hline
\end{tabular}

Stage 3: Group Four -Supervisor: Amira- Final Report "Jan. -2012"

\begin{tabular}{cccccccccccc}
\hline 1 & Soliman & 1.6 & 1.6 & 1.6 & 1.4 & 1.3 & 2 & 2 & 1.2 & 1.5 & 1.5 \\
\hline 2 & Faisal & 1.7 & 1.5 & 2 & 1.3 & 1.6 & 1.5 & 1.2 & 1.5 & 1.7 & 1.6 \\
\hline 3 & Turki & 2 & 2 & 1.5 & 2 & 1.4 & 2 & 2 & 1.5 & 2 & 1.8 \\
\hline 4 & Ibrahim & 1.9 & 1.9 & 2 & 2 & 1.5 & 1.5 & 2 & 2 & 2 & 1.9 \\
\hline Criteria: & 1: Improved & 2: Do the action with help & 3: Needs much help \\
\hline
\end{tabular}




\subsection{Final Results}

- Record the progress before and after using BG cubes, shapes, color therapy, in addition to the window designs, then compare the results.

- Performing Final Results.

- All results should be discussed and clarified over data conclusion.

\subsubsection{Data analysis}

Analyzing the data, will be through compare all the results before and after the experiment, and analysis the data using Excel and SPSS programs.

\subsubsection{Questionnaire (Checklist)}

Checklists are the appropriate one to measure the progress of the students' performance with the daily observation sent by the specialist. The First test was the TTest It was used to measure the effect calculators.

Figure (13) Graph showing comparison of mean score before and after on Stage 1

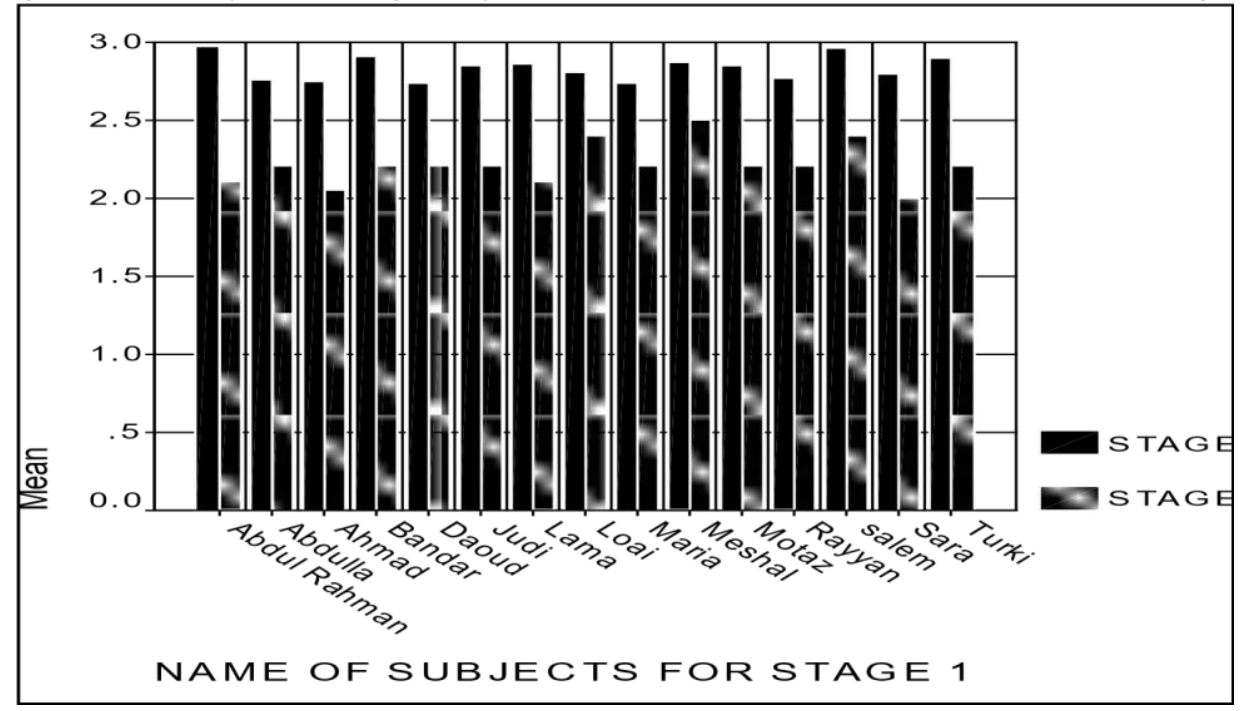

Figure (14) Graph showing comparison of mean score before and after on Stage 2

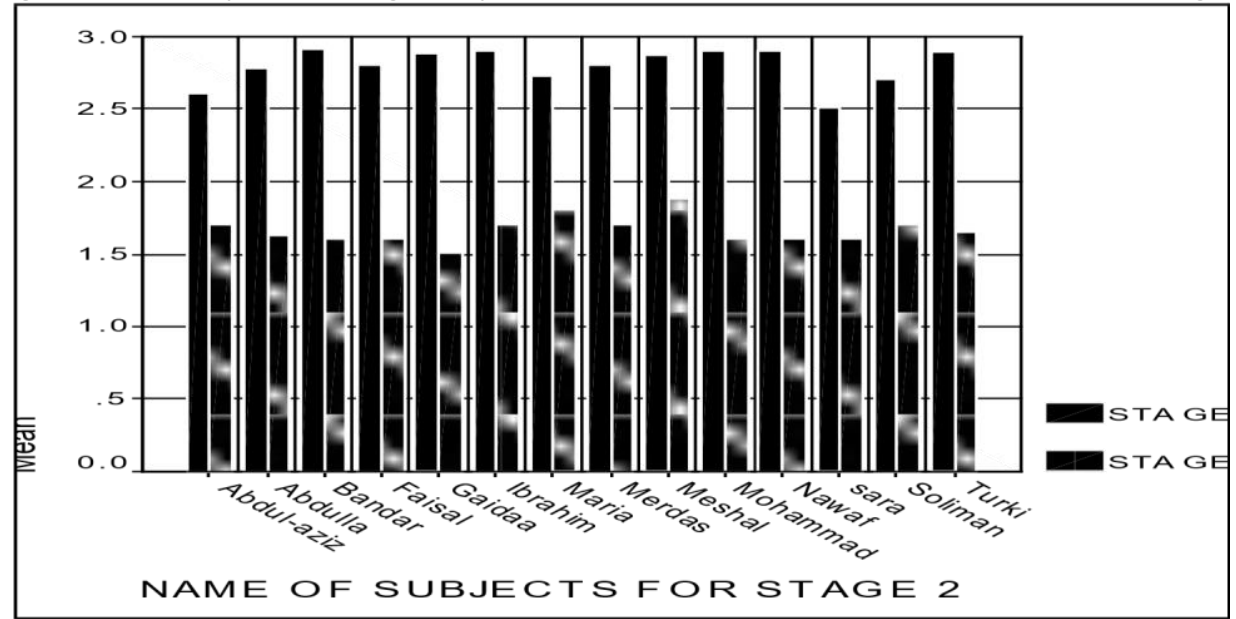




\section{ANOVA Test}

Table (10) ANOVA Test shows experiments final data analysis

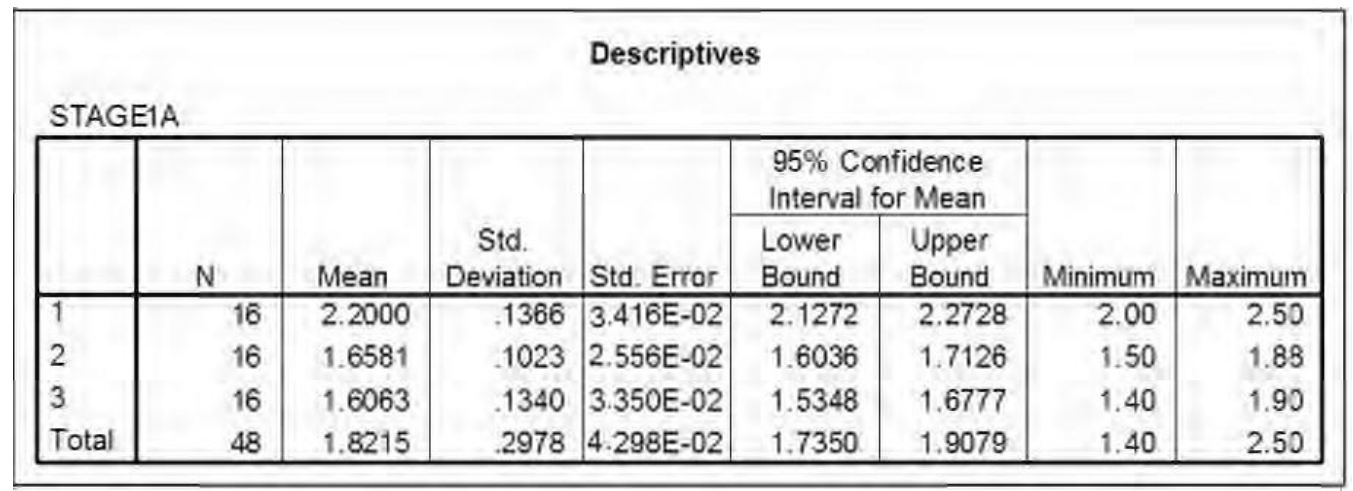

Table (11) ANOVA shows difference in means

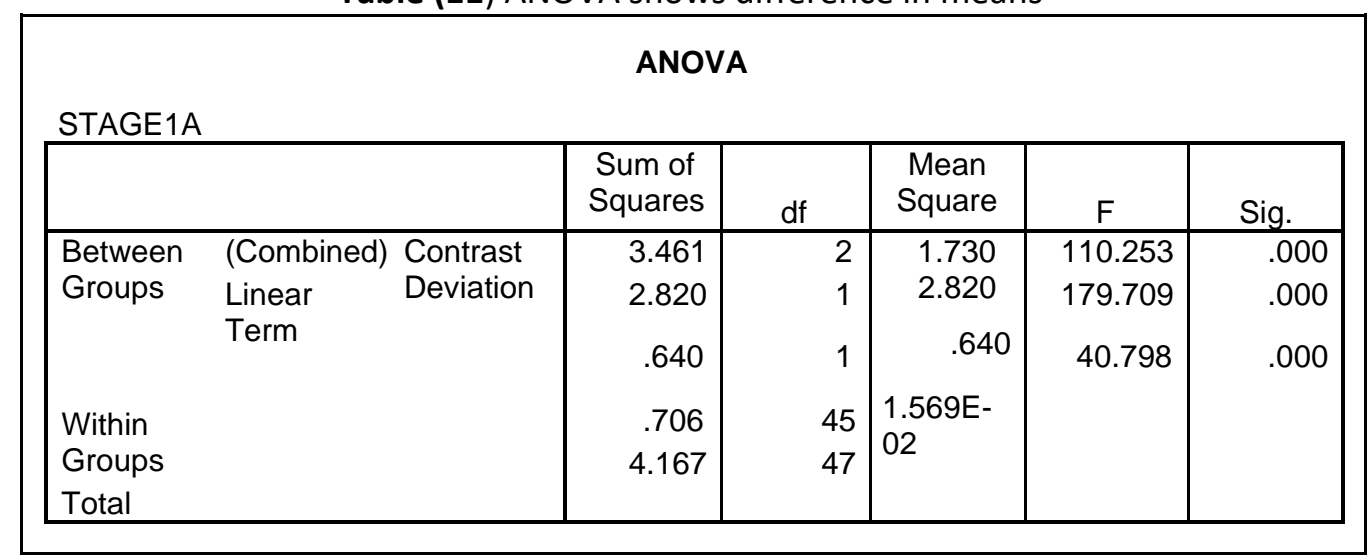

Table (12) Post Hoc Tests

\section{Multiple Comparisons}

Dependent Variable: STAGE1A

Tukey HSD

\begin{tabular}{|c|c|c|c|c|c|c|}
\hline \multirow[b]{2}{*}{ (I) SUB\# } & \multirow[b]{2}{*}{ (J) SUB\# } & \multirow[b]{2}{*}{$\begin{array}{c}\text { Mean } \\
\text { Difference } \\
(\mathrm{I}-\mathrm{J})\end{array}$} & \multirow[b]{2}{*}{ Std. Error } & \multirow[b]{2}{*}{ Sig. } & \multicolumn{2}{|c|}{$\begin{array}{l}\text { 95\% Confidence } \\
\text { Interval }\end{array}$} \\
\hline & & & & & $\begin{array}{l}\text { Lower } \\
\text { Bound }\end{array}$ & $\begin{array}{l}\text { Upper } \\
\text { Bound }\end{array}$ \\
\hline \multirow[t]{2}{*}{1} & 2 & $.5419^{*}$ & $4.429 E-02$ & .000 & .4345 & .6492 \\
\hline & 3 & $.5937^{*}$ & 4.429E-02 & .000 & .4864 & .7011 \\
\hline \multirow[t]{2}{*}{2} & 1 & $-.5419^{*}$ & 4.429E-02 & .000 & -.6492 & -.4345 \\
\hline & 3 & 5.187E-02 & 4.429E-02 & .476 & $-5.55 E-02$ & $.159 \varepsilon$ \\
\hline \multirow[t]{2}{*}{3} & 1 & $-.5937^{*}$ & 4.429E-02 & .000 & -.7011 & -.4864 \\
\hline & 2 & $-5.187 E-02$ & 4.429E-02 & .476 & -.1592 & $\begin{array}{l}5.547 \mathrm{E}- \\
02\end{array}$ \\
\hline
\end{tabular}

*. The mean difference is significant at the .05 level. 
Table (13) Homogeneous Subsets

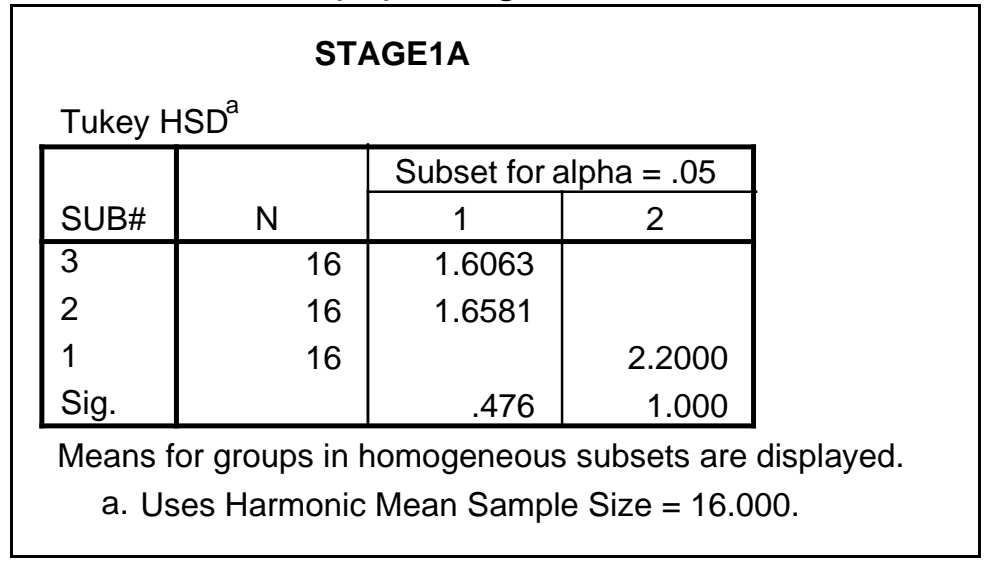

Table (14) Univariate Analysis of Variance

\begin{tabular}{|l|l|r|r|}
\hline \multicolumn{4}{|c|}{ Descriptive Statistics } \\
\hline \multicolumn{4}{|c|}{ Dependent Variable: STAGE1A } \\
\hline SUB\# & Mean & $\begin{array}{c}\text { Std. } \\
\text { Deviation }\end{array}$ & $\mathrm{N}$ \\
\hline 1 & 2.2000 & .1366 & 16 \\
2 & 1.6581 & .1023 & 16 \\
3 & 1.6063 & .1340 & 16 \\
Total & 1.8215 & .2978 & 48 \\
\hline
\end{tabular}

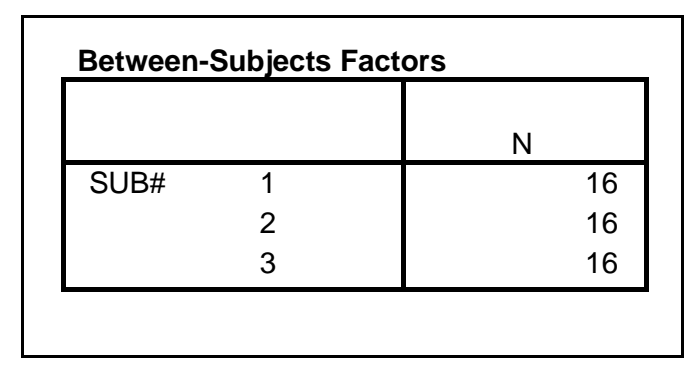

\begin{tabular}{|c|c|c|c|c|c|c|}
\hline \multicolumn{7}{|c|}{ Tests of Between-Subjects Effects } \\
\hline \multicolumn{7}{|c|}{ Dependent Variable: STAGE1A } \\
\hline Source & $\begin{array}{l}\text { Type III } \\
\text { Sum of } \\
\text { Squares }\end{array}$ & df & $\begin{array}{c}\text { Mean } \\
\text { Square }\end{array}$ & $\mathrm{F}$ & Sig. & $\begin{array}{c}\text { Eta } \\
\text { Squared }\end{array}$ \\
\hline Corrected Model & $3.461^{\mathrm{a}}$ & 2 & 1.730 & 110.253 & .000 & .831 \\
\hline Intercept & 159.250 & 1 & 159.250 & 10147.358 & .000 & .996 \\
\hline SUB\# & 3.461 & 2 & 1.730 & 110.253 & .000 & .831 \\
\hline Error & .706 & 45 & 1.569E-02 & & & \\
\hline Total & 163.417 & 48 & & & & \\
\hline Corrected Total & 4.167 & 47 & & & & \\
\hline
\end{tabular}

a. $\mathrm{R}$ Squared $=.831$ (Adjusted R Squared $=.823$ )

Overall, we may conclude that there is a significant difference between the score for Stage 1 and 2 , and more between Stage 1 and Stage 3 .

\subsection{Discussion}

The clear framework and methodology "design approach" that can help designers either architects or interiors to deal with the zones of energy in the interior architectural spaces, through the application of Bio Geometry Science in the interior architectural spaces, the study followed the checklists steps and methodology. 


\subsubsection{Detecting space energy (Diagnoses checklist)}

The aim is to detect and record the harmful Geo-pathic stress, measure the negative and positive energy grids, lines, and EMF in the interior spaces.

Table (15) Detecting space energy (Diagnoses checklist)

\begin{tabular}{|c|c|c|c|c|}
\hline No & Examines & Achieved & $\begin{array}{c}\text { Not } \\
\text { achieved }\end{array}$ & Remarks \\
\hline 1 & $\begin{array}{l}\text { Study the site plan (Google Earth) and } \\
\text { surrounding environment to check the electric } \\
\text { towers or high voltage lines. }\end{array}$ & $*$ & & \\
\hline 2 & $\begin{array}{l}\text { Sketch or use the building's floor plan to clarify } \\
\text { the harmful Geo-pathic stress. }\end{array}$ & $*$ & & \\
\hline 3 & $\begin{array}{l}\text { Identify the chosen space location with } \\
\text { reference to the north arrow. }\end{array}$ & $*$ & & \\
\hline 4 & Study the plan's shape and position. & * & & \\
\hline 5 & $\begin{array}{l}\text { Use the IKU and / or BG3 pendulums to sense } \\
\text { the energy. }\end{array}$ & $*$ & & \\
\hline 6 & Pendulums swing inward (clockwise) & $*$ & & $\begin{array}{c}\text { Indicates Positive } \\
\text { energy }\end{array}$ \\
\hline 7 & Pendulums swing outward (anticlockwise) & $*$ & & $\begin{array}{l}\text { Indicates } \\
\text { Negative energy }\end{array}$ \\
\hline 8 & $\begin{array}{l}\text { Use the convective (virtual cone) to examine } \\
\text { the negative energy lines and grids. }\end{array}$ & $*$ & & \\
\hline 9 & $\begin{array}{l}\text { Apply all the resulted lines and grids on the floor } \\
\text { plan. }\end{array}$ & 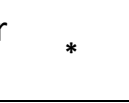 & & \\
\hline & $\begin{array}{l}\text { Identify the EMF sub panels, switches, light } \\
\text { units, appliances, and electric points. }\end{array}$ & $*$ & & ० \\
\hline 11 & $\begin{array}{l}\text { Record the EMF's negative energy by the } \\
\text { pendulums. }\end{array}$ & $*$ & & \\
\hline 12 & $\begin{array}{l}\text { Windows and door size, design, and } \\
\text { placement. }\end{array}$ & $*$ & & \\
\hline 13 & $\begin{array}{l}\text { Identify the negative energy that enters from } \\
\text { doors and windows. }\end{array}$ & $*$ & & \\
\hline 10 & & & & \\
\hline 14 & $\begin{array}{l}\text { Examine the impact of the space color on } \\
\text { the space and the } * \text { orientation). }\end{array}$ & \multicolumn{3}{|c|}{ humanity (Considering } \\
\hline
\end{tabular}

Achieved: 14

Score $90 \%$ or More: The design approach succeeds Score 75\% to $90 \%$ : Needs Improvement Score $75 \%$ to $50 \%$ : Failed 


\subsubsection{Correcting the negative energy (Correcting checklist)}

The aim is to correct the harmful Geo-pathic stress, measure the negative and positive energy grids, lines, and EMF by using the BG cubes and shapes, doors balance, and window designs.

Table (16) Correcting the negative energy (Correcting checklist)

\begin{tabular}{|c|c|c|c|c|}
\hline No & Examines & Achieved & $\begin{array}{c}\text { Not } \\
\text { achieved }\end{array}$ & Remarks \\
\hline 1 & $\begin{array}{l}\text { Study the best colors suit each case (Yellow\& } \\
\text { Purple) }\end{array}$ & $*$ & & \\
\hline 2 & $\begin{array}{l}\text { Examine the color impact on human using the } \\
\text { pendulums. }\end{array}$ & $*$ & & \\
\hline 3 & $\begin{array}{l}\text { Identify the color distribution in the } \\
\text { architectural spaces. }\end{array}$ & 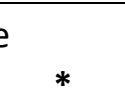 & & \\
\hline 4 & $\begin{array}{l}\text { Apply color in the architectural spaces that } \\
\text { need to be balanced. }\end{array}$ & $*$ & & \\
\hline 5 & IKU pendulum swing inward (clockwise) & $*$ & & $\begin{array}{l}\text { Indicates Positive } \\
\text { energy }\end{array}$ \\
\hline 6 & IKU pendulum swing outward (anticlockwise) & $*$ & & $\begin{array}{l}\text { Indicates } \\
\text { Negative energy }\end{array}$ \\
\hline 7 & $\begin{array}{l}\text { Use the convective (virtual cone) to examine } \\
\text { the negative energy lines and grids. }\end{array}$ & $*$ & & \\
\hline 8 & Use three of BG cubes to cover the whole area & $*$ & & \\
\hline 9 & Cleaning the BG cubes twice a week. & $*$ & & \\
\hline 10 & Daily recharge for the cubes on it is tray $5 \mathrm{~min}$. & $*$ & & \\
\hline 11 & $\begin{array}{l}\text { Use the stripes on the electric appliances, } \\
\text { Electric device wire, and the main electric box }\end{array}$ & $*$ & & \\
\hline 12 & $\begin{array}{l}\text { Design and apply new forms for windows and } \\
\text { monitor the progress. }\end{array}$ & $*$ & & \\
\hline 13 & Measure the energy in the spaces again. & $*$ & & \\
\hline
\end{tabular}

Achieved: 13

Score $90 \%$ or More: The design approach succeeds Score 75\% to $90 \%$ : Needs Improvement

Score $75 \%$ to $50 \%$ : Failed

From the previous analysis and design approach steps that relatively has been implemented in the case study and showed the most important way to investigate the energy and dealing with it in the architectural spaces.

\section{T-Test to compare all results for the 4 groups Before and after at each stage:}

\section{T-Test (Stage 1 before and after)}

Cohen's d = 5.3492

Effect size $r=0.9366$

Result: both show large effect 


\section{T-Test (Stage 2 before and after)}

Cohen's d $=7.84909$

Effect size $r=0.9690$

Result: Both show very large effects

T-Test (stage 3 before and after)

Cohen's $d=6.964$

Effect size $r=0.9611$

Result: According to the previous results the study came up with it was clear that both results show a very large effect happened to the children which means improvement in their performance and health

\section{T-Test (Stage 1 after and stage 2 after)}

Cohen's d $=4.49057$

Effect size $r=0.91349$

Both show very large effect

\section{T-Test (Stage 2 and 3 after)}

Cohen's $d=.43369$

Effect size $r=0.2119217$

Not, very large e, just medium effect

\section{ANOVA Test:}

Table (11) : ANOVA Showed that there is a difference in means

Table (14) : Univariate Analysis of Variance

Result: Very large partial eta value shows large effect size.

Thus it is clear that the research proofed the hypothesis that assumed behavioral and biological changes in humans, as a result of the interior space design changes and energy, in addition to raise in the efficiency of human health and performance, to assist in healing from diseases caused by interior architectural spaces energy.

Therefore since the applied study proofed that student performance had improved between stage 1 and 2 , and had a medium impact between 2 and 3 stages that means the study achieved its goals, by using the principles and theories of BioGeometry in the interior architectural spaces, to achieve the best results to maintain human health and the efficiency of performance and modify behavior.

\section{CONCLUSIONS AND RECOMMENDATIONS}

\subsection{Research Conclusions}

- Significance of the presence of energy in the interior architectural space and importance of increasing awareness of the energy impact on human beings in the interior architectural space. 
- Identifying a number of sciences and knowledge of energy, with the advancement in instrumentation of qualitative energy led to understand this energy and the new discoveries of unconsciously in architecture and interior design of the builtin environments.

- The presence of many factors and several impacts on humans in the system of interior architectural space, and leads to unbalance at all levels and therefore was able to put the first

- Bio Geometry can cure any deficiency and unbalance in energy, to help in achieving comfort and health in the architectural spaces.

- Some colors provide comfort in the interior architectural spaces. And had an impact on humans' psychological state and activities causes relax or movement. It also have another impact on the physical state that causes discomfort. - ADHD is common especially among school children; one of the reasons is the interior space's negative energy. It can be healed by using the design approach set by the research to achieve the energy balance in the architectural space.

\subsection{Recommendations}

- Geologists should prepare detailed maps for cities using the satellite to identify the black streams and geological faults, to be as a guide that helps the designer in the design process to produce building matches with the environment. - Link the sciences and theories of interior and architectural design, with the other sciences such as bio-geometry, which deals with human energy in the architectural spaces that will help the designer to achieve the human needs into those spaces and raise their performance.

- Designers can use the clear framework and methodology done in the research as a design approach, through which the designers either architect or interior can deal with the zones of energy in the interior architectural spaces to improve the human health and performance in those spaces.

- Designers should concentrate on the relationship between the shape and configuration as the basis for the BG science, in order to achieve balance and harmony between human body as an energy field, and the energy of the interior space in addition to use the color.

\section{References}

Baker, Paula-and others-"A Healthy House", 2001

Dr. Ibrahim Karim-"What is energy-Back to the Future"-International Union of Architects Conference- Bibliotheca Alex.- Alexndrina, 2002

Ibrahim Karim," Bio-Geometrical Energy Balancing" cd, 2002

Ibrahim Karim-"Back To A future For Mankind"-publisher: Bio-geometry consultant centre, 2009 http://www.adhd.com/index.html

http://www.biogeometry.com 